\title{
Mask-RCNN generalisation to vectorise surface-type objects of old maps
}

\author{
V. Árvai ${ }^{\mathrm{a}}, \mathrm{M}$. Gede $^{\mathrm{a}}$ \\ ${ }^{a}$ Institute of Cartography and Geoinformatics, ELTE Eötvös Loránd University, Budapest - valentin356@gmail.com, \\ saman@map.elte.hu
}

Keywords: Automatic vectorisation, Deep learning, Mask-RCNN, Archive maps

\begin{abstract}
:
Raster to vector conversion is a very common task in geoinformatics. Traditionally it is performed through manual digitizing. Because of its nature, it takes a lot of time to vectorise a scanned map, usually hours/days. However, there are plenty of commercial and non-commercial software, that has a built-in automatic vectorisation module. These modules can be used to digitise maps produced using the latest GIS software. For old maps, these modules are not always usable. The reason is usually that the map was produced using older technology (e.g. hand-drawn symbols or text) or is simply in poor quality.
\end{abstract}

As there is no general method to automate the vectorisation of archive maps, we have focused on this problem. However, there are several studies on the subject (which are processes mostly newer maps). Most of them use a colour filtering-based method mixed with various morphological filters. This method was tested in a previous study on the archive map sheet of our choice (Gede, Árvai, Vassányi, \& et al., 2020). The results showed that certain line-type objects can be vectorised, but most features cannot be extracted with simple colour filters and most of them are too complex to be delimited with common (same cluster) rules.

To solve this problem, we started working on another solution, based on Mask-RCNN (He, Gkioxari, Dollár, \& Girshick, 2017). Mask-RCNN is a deep learning-based module that predicts the segmentation mask inside the features bounding box. It extends Faster R-CNN, which is based on a convolutional neural network (extended with RPN). The base code we used is Matterport's implementation, which using the transfer learning method (ResNet101 layer structure). It takes annotated training images as input, in which the features are delimited. This module works with a lot of images, so it needs decent hardware. Because of that, we used Google Colaboratory's GPU-accelerated hardware environment.

The process consists of two main parts: pre-processing and model creation. In the pre-processing phase, the image cuts were created and annotated. In the model creation phase the model's properties were declared, such as batch size, iteration count etc...

In our generalisation of Mask-RCNN, we have used sheets of the Third Austro-Hungarian Military Survey. These sheets are very noisy and have several printing issues (e.g. blue overprint on black ink). To increase detection accuracy of the model, objects are separated by geometry type. In this study, we only discuss the surface type objects. Based on the map's legend, this includes settlements, water surfaces, forested areas, and orchards.

Filters were used as well, to improve the quality of the input images and the detection accuracy. Additional extensions were created too, to automatize some parts of the pre-processing phase of the training images, as well as we added support to save the output in georeferenced vector format (.shp).

With the additional modules we achieved $80 \%$ accuracy. False-positive detections are under 5\%, most of the cases it is zero. Although the object delineation is not perfect in some cases, this can be improved with additional filtering procedures. The aim is to further improve the delineation and the accuracy of the detection. For this, we test a bunch of new filters, and create a new processing layer order with the tested filters. Also, the original map's pre-processing phase will be revised, to improve the quality of the input image cuts by surface type.

The other main objective of the project is to make the created process applicable to other maps (new or old) by modifying the input data and making minor changes. This would reduce the time needed for the time-consuming vectorisation process. Of course, the first half of the process is relatively time consuming (image annotation), but for the vectorisation of a series of maps, this time is still negligible in the overall context.

\section{Acknowledgement:}

EFOP-3.6.3-VEKOP-16-2017-00001: Talent Management in Autonomous Vehicle Control Technologies. The Project is supported by the Hungarian Government and co-financed by the European Social Fund. 